\title{
Structural Basis of the Interaction Between Ubiquitin Specific Protease 7 and Enhancer of Zeste Homolog 2
}

\author{
A Bojagora ${ }^{1}$ \\ ${ }^{1}$ York University, Toronto, Ontario \\ anna.bojagora@gmail.com
}

\begin{abstract}
Anna Bojagora, Varvara Gagarina, Ira Kay Lacdao, Niharika Luthra, Roland Pfoh, Sadaf Mohseni, Danica Chaharlangi, Nadine Tan and Vivian Saridakis Department of Biology, York University, 4700 Keele Street, Toronto, Ontario, M3J1P3, Canada The deubiquitinating enzyme, USP7, regulates the turnover of proteins involved in many diverse cellular processes, including maintenance of genome stability, tumour suppression, epigenetics, DNA replication, cell division, and immune response. Previously defined USP7 substrates, including GMPS, UHRF1, and ICP0, were characterized to interact with the C-terminal domain of USP7 via a KxxxK motif. We identified a common motif in Enhancer of Zeste 2 (EZH2), a histone methyltransferase and the catalytic component of the Polycomb Repressive Complex 2 (PRC2). PRC2 is responsible for methylating Histone 3 Lys27 (H3K27) leading to transcriptional repression of genes involved in differentiation and development. The interaction between USP7 and EZH2 was demonstrated by GST pull-down and co-immunoprecipitation experiments. We co-crystallized Ubl123 of USP7 with an EZH2 peptide containing the predicted interaction site and characterized the structural basis of the interaction, identifying the key residues involved. Mutagenesis studies of these residues demonstrated abolished binding between USP7 and EZH2. The functional relationship between USP7 and EZH2 was investigated using USP7 knock-down and knock-out experiments which corresponded with reduced EZH2 levels in HCT116 carcinoma cells and decreased H3K27Me3 levels in HCT116 USP7 knockout cells respectively. These findings reveal that USP7 regulates both the stability and function of EZH2.
\end{abstract}

Acta Cryst. (2020). A76, a112 